\title{
Analytical inverse kinematics for 5-DOF humanoid manipulator under arbitrarily specified unconstrained orientation of end-effector Masayuki Shimizu*
}

\author{
Department of Mechanical Engineering, Shizuoka University, Hamamatsu, Japan
}

(Accepted February 8, 2014. First published online: March 13, 2014)

\begin{abstract}
SUMMARY
This paper proposes an analytical method of solving the inverse kinematic problem for a humanoid manipulator with five degrees-of-freedom (DOF) under the condition that the target orientation of the manipulator's end-effector is not constrained around an axis fixed with respect to the environment. Since the number of the joints is less than six, the inverse kinematic problem cannot be solved for arbitrarily specified position and orientation of the end-effector. To cope with the problem, a generalized unconstrained orientation is introduced in this paper. In addition, this paper conducts the singularity analysis to identify all singular conditions.
\end{abstract}

KEYWORDS: Inverse kinematics; 5-DOF manipulator; Unconstrained orientation; Singular configuration; Humanoid robots.

\section{Introduction}

General robotic manipulators have six or more joints, since six degrees-of-freedom (DOFs) are required to locate the end-effector at any position and orientation in space. Therefore, solving the inverse kinematic problem for such manipulators has been intensively studied over several decades (e.g., refs. [1-6]). To date, various analytical as well as numerical methods for inverse kinematic computation have been developed.

Although a manipulator involving six or more joints is versatile, the six or more DOF manipulator is not always necessary for performing a task. A well-known example is the arc welding task. Since any rotation about the center line of the welding torch does not affect the task, a 5-DOF manipulator is sufficient to accomplish the task. Thus, even manipulators with less than six joints are usable for achieving practical tasks.

This paper addresses the inverse kinematic problem for a 5-DOF manipulator. This work is motivated by an attempt to accomplish practical tasks by using a small humanoid robot. The robot, shown in Fig. 1, consists of two 6-DOF legs, two 4-DOF arms, and 1-DOF waist. Since all the leg joints are devoted to keeping the standing balance of the robot, the dimension of the workspace of a single arm's tip is less than six. Since the waist joint can be employed to augment the dimension of the workspace of the single arm tip, this paper considers exploiting the waist joint to maximize the workspace dimension. Hence, the resultant workspace dimension of the single arm is five. Note that since the waist joint is shared with both arms, the waist joint is dedicated to a single arm only. Hence, this paper assumes that only a single arm is used for task execution, and the remaining arm is not used. Thus, an appropriate inverse kinematic computation method is required for the 5-DOF manipulator to carry out the tasks.

\subsection{Related work}

The inverse kinematic problem for serial manipulators with five or less DOFs has been addressed by a number of researchers. Sugimoto and Duffy ${ }^{7}$ analyzed the kinematics of a general 5-DOF manipulator

* Corresponding author. E-mail: tmsimiz@ipc.shizuoka.ac.jp 


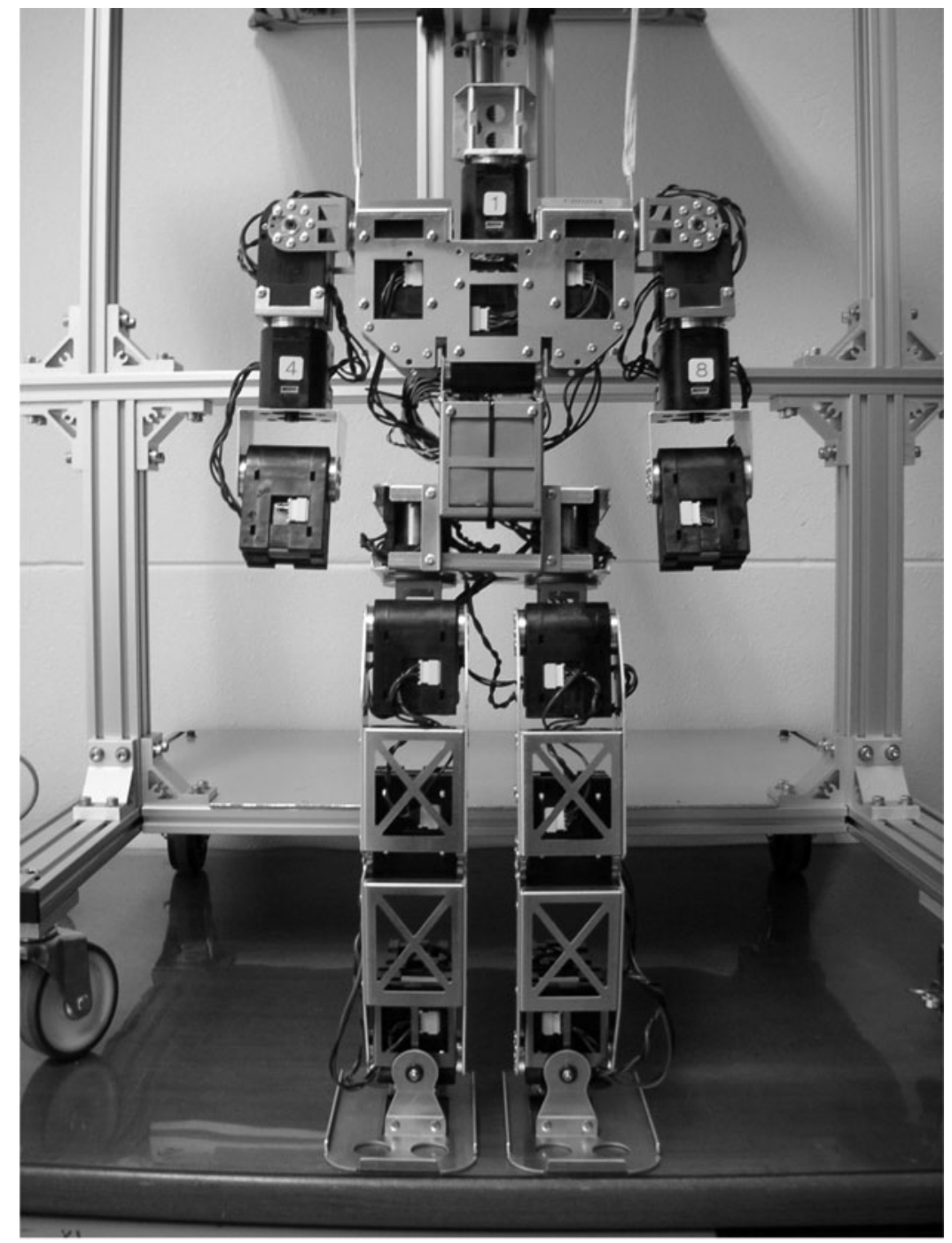

Fig. 1. Humanoid robot "Choromet2".

by introducing hypothetical joints and links. Tsai and Morgan ${ }^{8}$ proposed a numerical approach to solving the inverse kinematics for a general $6 \mathrm{R}$ or $5 \mathrm{R}$ manipulator by using continuation methods. Angeles ${ }^{9}$ employed the least-squares approximation technique to solve the inverse kinematics of general 5-DOF manipulators in a numerical way.

Manseur and Doty ${ }^{10}$ showed that the inverse kinematic solution for a general $4 \mathrm{R}$ manipulator is unique and can be analytically derived in a closed-form. In contrast, they also revealed that there are special structures. If a manipulator includes such a special structure, the inverse solution is not unique. One of the special structures is the wrist structure, where three consecutive joint axes intersect at a point. This wrist structure is incorporated in most commercial manipulators. Interestingly, this implies that solving inverse kinematics for a commercial manipulator may be more complicated than for a general manipulator without the special structures. Using the closed-form solution for the $4 \mathrm{R}$ manipulator, they also developed an efficient numerical method to compute an inverse kinematic solution for a general $5 \mathrm{R}$ manipulator. ${ }^{11}$

Zhou et al. ${ }^{12}$ proposed a vector algebraic approach to representing kinematic equations. With this method, they derived an eighth order polynomial equation as a closed-form solution for a general $5 R$ manipulator. In general, however, the eighth order polynomial cannot be solved analytically. Later, Zhou and $\mathrm{Xi}^{13}$ showed that the inverse kinematic solution can be described by a set of first order polynomials for a general $5 \mathrm{R}$ manipulator without special geometries. 


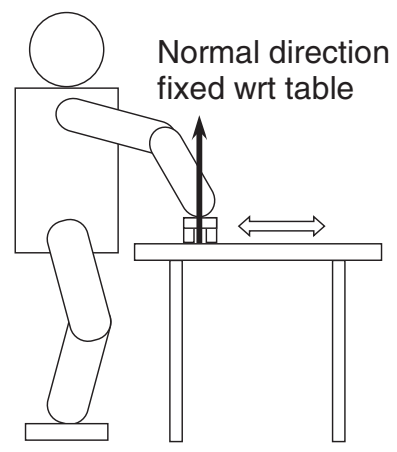

Fig. 2. Table wiping task.

Chen and $\mathrm{GaO}^{14}$ developed a computer program which provides closed-form inverse solutions for a given nonredundant manipulator. Their method relies on the existence of a special geometry in the manipulator structure, hence not all types of manipulators can be treated by their method.

For several commercial and special 5-DOF manipulators, closed-form inverse solutions have been obtained. Goel ${ }^{15}$ developed a closed-form solution for the AdeptOne robot, which is a SCARAtype 5-DOF manipulator. For the Pioneer 2 arm, several researchers have tackled to obtain closedform solutions and to identify singular configurations. ${ }^{16,17,18}$ Wang and Ishimatsu ${ }^{19}$ analyzed the kinematics of a 5-DOF prosthetic arm that has a special shoulder mechanism. Wang ${ }^{20}$ analytically solved the inverse kinematics of a 5-DOF arm attached to an amusement robot to identify the reachable position and orientation of the $\operatorname{arm}^{1}$.

\subsection{Problem statement}

The aim of this work is to carry out practical tasks by a 5-DOF manipulator, which is composed of the waist and a single arm of the humanoid robot shown in Fig. 1. Specifically, one of the target tasks is to wipe a table with a cloth, as shown in Fig. 2. Moreover, this project attempts to achieve not only the table wiping task but also other similar tasks, such as window cleaning, cleaning a whiteboard with an eraser, and ironing clothes on an ironing board.

Recently, Sato et al. ${ }^{21}$ performed a whiteboard cleaning task by a small humanoid robot equipped with a 4-DOF arm. They attached a 2-DOF special passive end-effector to the arm's tip in order to compensate insufficient DOFs, because they considered that a 6-DOF arm is required for performing the task. However, actually, a 5-DOF arm suffices to perform the task, since the task is not thwarted by any rotation about the normal axis of the table or whiteboard. Therefore, the special device is not required if a 5-DOF arm is available. In this paper, no special device is used, since the DOF of the arm is five.

To achieve the table wiping task, we have to solve a special inverse kinematic problem for the 5-DOF manipulator. More specifically, we have to determine five joint displacements so that given target position and orientation of the end-effector are ensured except a rotation about the normal axis of the tabletop. In the following, this axis and its direction are referred to as an unconstrained axis and unconstrained direction, respectively. The difficulty in solving the problem is that the unconstrained direction is fixed with respect to the environment. Furthermore, the direction varies depending on the task and environment. For example, the unconstrained direction will be vertical when wiping a table on the floor, whereas it may be horizontal for window cleaning.

The conventional inverse kinematic solving methods for a 5-DOF manipulator are not applicable to the above problem. Some methods ${ }^{12,13,14}$ request that reachable position and orientation of the end-effector be given by the user. However, the reachable position and orientation are not easily found for the 5-DOF manipulator, as discussed in ref. [20]. In some other methods, ${ }^{7,8,18}$ the unconstrained

\footnotetext{
${ }^{1}$ In this paper, the reachable position and orientation means that the position and orientation is actually achievable by the manipulator. In contrast, the desired position and orientation means that the position and orientation may not be achieved, though the user wishes to achieve it.
} 
axis is fixed with respect to the end-effector, hence it varies as the manipulator changes the endeffector's orientation. Although the unconstrained direction is fixed with respect to the environment in several methods, ${ }^{15,16,17,20}$ the direction is absolutely fixed, and the user cannot change it. Since the unconstrained direction is dependent on the task and environment, the direction should be variable.

To clarify why the conventional methods do not work, let us briefly consider how to solve the inverse kinematic problem. Let $\chi_{i}(i=1, \ldots, 6)$ be independent parameters representing the endeffector's position and orientation. Also, let $\theta_{j}(j=1, \ldots, 5)$ be the joint displacements of the 5-DOF manipulator. Assume that the forward kinematic equations are given by

$$
\chi_{i}=f_{i}\left(\theta_{1}, \ldots, \theta_{5}\right), \quad(i=1, \ldots, 6) .
$$

Notice that the number of the kinematic equations is six, while that of the joint displacements is five.

To solve this problem, two approaches can be taken. One is to reduce the number of the kinematic equations, while the other is to increase the number of the joint displacements. In the former approach, for example, refs. [15-17, 20], one of the kinematic equations is neglected, and the remaining five simultaneous equations are solved. However, the derived solution does not satisfy the neglected kinematic equation, if the given set of $\chi_{i}$ is kinematically unreachable by the manipulator. Thus, this approach may not provide the feasible inverse solution. In the latter approach, for example, refs. [7, 8,18 ], a virtual or hypothetical joint is added to the manipulator, and the six simultaneous equations are solved.

To see this more specifically, suppose that the virtual joint is added to the kinematic equations Eq. (1):

$$
\chi_{i}=f_{i}\left(\theta_{1}, \ldots, \theta_{5}, \theta_{v}\right), \quad(i=1, \ldots, 6)
$$

where $\theta_{v}$ denotes the virtual joint displacement. Furthermore, suppose that the solution to this simultaneous equations is given by

$$
\begin{aligned}
& \theta_{j}=g_{j}\left(\chi_{1}, \ldots, \chi_{6}\right), \quad(j=1, \ldots, 5) \\
& \theta_{v}=g_{v}\left(\chi_{1}, \ldots, \chi_{6}\right) .
\end{aligned}
$$

Notice that the virtual joint displacement $\theta_{v}$ should have a fixed value, denoted as $\theta_{v 0}$, because the virtual joint is immobilized. Thus, we have to solve the nonlinear equation

$$
g_{v}\left(\chi_{1}, \ldots, \chi_{6}\right)-\theta_{v 0}=0
$$

to obtain the feasible solution.

Let $\phi$ be the rotation angle about the unconstrained axis. Since $\phi$ is completely represented by $\chi_{i}$ $(i=1, \ldots, 6)$, it is described in the function form

$$
\phi=h\left(\chi_{1}, \ldots, \chi_{6}\right) .
$$

From this, we can represent $\chi_{i}(i=1, \ldots, 6)$ explicitly by introducing slack variables $\eta_{i}(i=$ $1, \ldots, 5)$ :

$$
\chi_{i}=h_{i}^{-1}\left(\eta_{1}, \ldots, \eta_{5}, \phi\right), \quad(i=1, \ldots, 6)
$$

Substituting (6) into (4), we have

$$
g_{v}\left(\eta_{1}, \ldots, \eta_{5}, \phi\right)-\theta_{v 0}=0 .
$$

Thus, $\phi$ must satisfy this constraint equation. Letting $\phi_{0}$ be the solution to the constraint equation, and substituting it into (6), $\chi_{i}$ can be represented by the slack variables $\eta_{i}$ only:

$$
\chi_{i}=h_{i}^{-1}\left(\eta_{1}, \ldots, \eta_{5}, \phi_{0}\right), \quad(i=1, \ldots, 6)
$$

Finally, substituting (8) into (3), we can obtain the feasible inverse solution. 


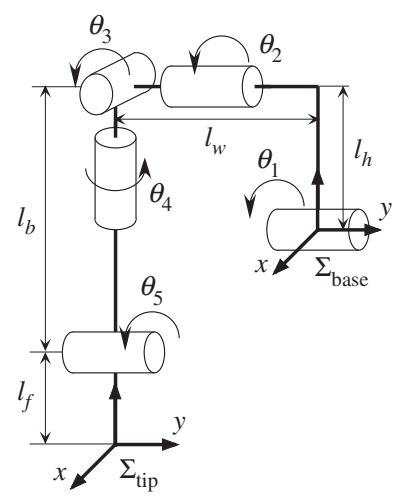

Fig. 3. 5-DOF manipulator model.

It is obvious that some difficulties lie in this approach. For example, it may be difficult to compute the inverse mapping $h_{i}^{-1}$ in (6) in an analytical way. Furthermore, it may be difficult or impossible to derive the analytical solution of the nonlinear Eq. (7). To avoid these difficulties, the unconstrained axis is deliberately aligned with the virtual joint axis in the previous methods. In addition, if the virtual joint is attached to the tip of the manipulator, $\theta_{v}$ is equal to $\phi$. Then, the inverse mapping $h_{i}^{-1}$ can be chosen as the direct one, namely, $\eta_{i}=\chi_{i}(i=1, \ldots, 5)$. This restriction drastically simplifies the problem, and the analytical solution has already been derived as shown in the previous methods. However, in the case that the unconstrained axis is not fixed and arbitrarily chosen, the conventional approach is not applicable.

\subsection{Objective of this paper}

The objective of this paper is to develop an analytical inverse kinematic computation methodology for a 5-DOF manipulator to achieve target position and orientation except an orientation about the unconstrained axis. A distinctive feature of this solving method is that the unconstrained direction can be arbitrarily specified with reference to the environment, which is impossible in the previous methods. In addition, this paper performs the singularity analysis to derive all singular conditions. Since the inverse kinematic problem depends on the unconstrained direction, the singular conditions also depend on it.

This paper is organized in the following manner. Section 2 describes the 5-DOF manipulator model and the inverse kinematic problem addressed in the paper. Section 3 computes the forward kinematics. Then, the inverse kinematic problem is solved in Section 4. In Section 5, the singular conditions are derived in an analytic way. Finally, the validity of the proposed method is verified by numerical examples in Section 6. The extension and limitation of the method is discussed in Section 7.

\section{Problem Definition}

\subsection{Manipulator model}

The kinematic structure of the 5-DOF manipulator handled in this paper is illustrated in Fig. 3. It is generated from the kinematic model of the waist and right arm of the humanoid robot shown in Fig. 1. In Fig. 3, all five joints are revolute, and three consecutive joint axes 2, 3, and 4 intersect at a point, which corresponds to the right shoulder of the humanoid robot. Hence, these three joints can be regarded as a virtual spherical joint. Note that the existence of a virtual spherical joint is the sufficient condition to guarantee the solvability of the inverse kinematics for a 5-DOF manipulator, as proven in ref. [11].

First, let us describe the manipulator structure. To this end, the Denavit-Hartenberg notation ${ }^{22}$ is employed. All links and joints are numbered according to the D-H convention. The base coordinate frame is placed on the base joint so that the $y$-axis coincides with the joint axis, and the $z$-axis directs upward, as shown in Fig. 3. Note that this base frame is not the link frame 0 defined by the D-H 
Table I. D-H parameters of 5-DOF manipulator model.

\begin{tabular}{ccccc}
\hline$i$ & $a_{i}$ & $\alpha_{i}(\mathrm{rad})$ & $d_{i}$ & $\theta_{i}(\mathrm{rad})$ \\
\hline 1 & $l_{h}$ & 0 & 0 & $\theta_{1}+\pi / 2$ \\
2 & 0 & $\pi / 2$ & $-l_{w}$ & $\theta_{2}+\pi$ \\
3 & 0 & $\pi / 2$ & 0 & $\theta_{3}-\pi / 2$ \\
4 & 0 & $\pi / 2$ & $-l_{b}$ & $\theta_{4}-\pi / 2$ \\
5 & 0 & $\pi / 2$ & 0 & $\theta_{5}+\pi$ \\
\hline
\end{tabular}

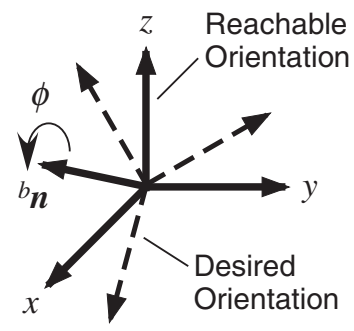

Fig. 4. Definition of $\phi$.

convention. The tip coordinate frame is attached to the tip so that all the frame axes are aligned with the corresponding axes of the base frame when the manipulator is at the default configuration, which is shown in Fig. 3. Note again that this tip frame is not the link frame 5 defined by the D-H convention. Then, the link coordinate frames are automatically defined based on the D-H rule.

In addition, the positive rotational directions of all joints are depicted in Fig. 3. The offsets of the joint angles are determined so that the manipulator is at the default configuration when all joint angles are zero. Finally, all the D-H parameters of the 5-DOF manipulator are obtained as listed in Table I.

\subsection{Formulation of inverse kinematic problem}

As mentioned in the previous section, the inverse kinematic problem addressed in this paper can be defined as follows: given target tip position and orientation, determine the joint displacements which satisfy the specified tip position and orientation except an orientation about an unconstrained axis fixed with reference to the environment.

Since the rotation about the unconstrained axis is free, it can be regarded as the augmented DOF of the manipulator, though it is not controllable. It should be noted that the augmented DOF cannot be replaced by a virtual joint. To incorporate the augmented DOF, the unconstrained rotation angle is regarded as an auxiliary joint variable. With this auxiliary variable, the number of the joint variables in the kinematic equations becomes six, which is same as the number of the independent kinematic equations. In this way, we can make the inverse kinematic problem solvable.

Let us formulate the inverse kinematic problem. Assume that the target tip position and orientation are given by the position vector ${ }^{b} \boldsymbol{p}_{t}^{d} \in \mathfrak{R}^{3}$ and the rotation matrix ${ }^{b} \boldsymbol{R}_{t}^{d} \in \mathrm{SO}(3)$, respectively. Assume also that the unconstrained direction is given by the unit vector ${ }^{b} \boldsymbol{n} \in \mathfrak{R}^{3}$. Note that the superscript on the left side of a vector/matrix denotes the reference coordinate frame in which the vector/matrix is described. In this paper, the symbol $b$ and $t$ indicate the base and tip coordinate frames respectively.

Now let us introduce the auxiliary variable, denoted by $\phi \in \Re$, which represents a rotation angle about the unconstrained axis. The role of this new variable is to adjust a given target orientation so that the adjusted orientation as well as the target position are reachable by the 5-DOF manipulator, as shown in Fig. 4. Suppose that the forward kinematic equations are given by the homogeneous transformation matrix ${ }^{b} \boldsymbol{T}_{t}(\boldsymbol{\theta}) \in \mathfrak{R}^{4 \times 4}$, where $\boldsymbol{\theta} \in \mathfrak{R}^{5}$ is the joint displacement vector. Then, the auxiliary variable $\phi$ as well as the joint displacements $\boldsymbol{\theta}$ have to be determined to satisfy the matrix equation

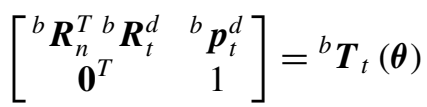


where ${ }^{b} \boldsymbol{R}_{n} \in \mathrm{SO}(3)$ is the rotation matrix, which accounts for the rotation by $\phi$ about the unconstrained axis ${ }^{b} \boldsymbol{n}$. It is important to notice that the given target orientation ${ }^{b} \boldsymbol{R}_{t}^{d}$ is adjusted by ${ }^{b} \boldsymbol{R}_{n}^{T}$ so that the resultant orientation ${ }^{b} \boldsymbol{R}_{n}^{T}{ }^{b} \boldsymbol{R}_{t}^{d}$ is reachable. According to the Rodrigues rotation formula, ${ }^{22}$ the rotation matrix ${ }^{b} \boldsymbol{R}_{n}$ is given by

$$
{ }^{b} \boldsymbol{R}_{n}=\cos \phi \boldsymbol{I}+\sin \phi\left[{ }^{b} \boldsymbol{n} \times\right]+(1-\cos \phi)\left[{ }^{b} \boldsymbol{n}^{b} \boldsymbol{n}^{T}\right]
$$

where $\boldsymbol{I} \in \mathfrak{R}^{3 \times 3}$ is the identity matrix, and $\left[{ }^{b} \boldsymbol{n} \times\right] \in \mathfrak{R}^{3 \times 3}$ is a skew-symmetric matrix composed of ${ }^{b} \boldsymbol{n}$, which is defined in ref. [23] as

$$
\left[{ }^{b} \boldsymbol{n} \times\right]=\left[\begin{array}{ccc}
0 & -n_{z} & n_{y} \\
n_{z} & 0 & -n_{x} \\
-n_{y} & n_{x} & 0
\end{array}\right]
$$

where ${ }^{b} \boldsymbol{n}=\left[\begin{array}{lll}n_{x} & n_{y} & n_{z}\end{array}\right]^{T}$.

Since ${ }^{b} \boldsymbol{p}_{t}^{d}$ and ${ }^{b} \boldsymbol{R}_{t}^{d}$ are given, the unknown variables in (9) are the joint displacements $\boldsymbol{\theta}$ and the unconstrained rotation angle $\phi$. Thus, the inverse kinematic problem can be formulated as the problem of computing $\boldsymbol{\theta}$ and $\phi$ to satisfy (9). Notice that, by introducing $\phi$, we do not need to guarantee the reachability of the target tip position and orientation. If they are unreachable, a nonzero $\phi$ is obtained as the inverse solution, and the quantity of $\phi$ indicates the difference between the target and reachable orientations.

\section{Forward Kinematics}

The forward kinematics of the manipulator shown in Fig. 3 is calculated from the D-H parameters given in Table I. The homogeneous transformation matrix between the adjacent link frames $i-1$ and $i$ is given in ref. [22] by

$$
{ }^{i-1} \boldsymbol{T}_{i}=\left[\begin{array}{cc}
{ }^{i-1} \boldsymbol{R}_{i}{ }^{i-1} \boldsymbol{p}_{i} \\
\mathbf{0}^{T} & 1
\end{array}\right]
$$

where

$$
\begin{aligned}
{ }^{i-1} \boldsymbol{R}_{i} & =\left[\begin{array}{ccc}
\cos \theta_{i} & -\sin \theta_{i} \cos \alpha_{i} & \sin \theta_{i} \sin \alpha_{i} \\
\sin \theta_{i} & \cos \theta_{i} \cos \alpha_{i} & -\cos \theta_{i} \sin \alpha_{i} \\
0 & \sin \alpha_{i} & \cos \alpha_{i}
\end{array}\right] \\
{ }^{i-1} \boldsymbol{p}_{i} & =\left[\begin{array}{lll}
a_{i} \cos \theta_{i} & a_{i} \sin \theta_{i} & d_{i}
\end{array}\right]^{T} .
\end{aligned}
$$

The homogeneous transformation matrix between the base frame and the link frame 0 is

$$
{ }^{b} \boldsymbol{T}_{0}=\left[\begin{array}{cc}
{ }^{b} \boldsymbol{R}_{0} & { }^{b} \boldsymbol{p}_{0} \\
\mathbf{0}^{T} & 1
\end{array}\right]=\left[\begin{array}{cccc}
-1 & 0 & 0 & 0 \\
0 & 0 & 1 & 0 \\
0 & 1 & 0 & 0 \\
0 & 0 & 0 & 1
\end{array}\right] .
$$

Similarly, the transformation between the link frame 5 and the tip frame is

$$
{ }^{5} \boldsymbol{T}_{t}=\left[\begin{array}{cc}
{ }^{5} \boldsymbol{R}_{t} & { }^{5} \boldsymbol{p}_{t} \\
\mathbf{0}^{T} & 1
\end{array}\right]=\left[\begin{array}{cccc}
1 & 0 & 0 & 0 \\
0 & 1 & 0 & 0 \\
0 & 0 & 1 & -l_{f} \\
0 & 0 & 0 & 1
\end{array}\right] .
$$


Using these homogeneous transformation matrices, the forward kinematic equations are described in the matrix form

$$
{ }^{b} \boldsymbol{T}_{t}={ }^{b} \boldsymbol{T}_{0}{ }^{0} \boldsymbol{T}_{1}{ }^{1} \boldsymbol{T}_{2}{ }^{2} \boldsymbol{T}_{3}{ }^{3} \boldsymbol{T}_{4}{ }^{4} \boldsymbol{T}_{5}{ }^{5} \boldsymbol{T}_{t} .
$$

Extracting the position ${ }^{b} \boldsymbol{p}_{t}$ and orientation ${ }^{b} \boldsymbol{R}_{t}$ of the tip frame from (15), we obtain

$$
\begin{aligned}
{ }^{b} \boldsymbol{p}_{t} & ={ }^{b} \boldsymbol{R}_{0}\left({ }^{0} \boldsymbol{R}_{1}{ }^{1} \boldsymbol{p}_{w s}+{ }^{0} \boldsymbol{R}_{4}{ }^{4} \boldsymbol{p}_{s e}+{ }^{0} \boldsymbol{R}_{5}{ }^{5} \boldsymbol{p}_{e t}\right) \\
{ }^{b} \boldsymbol{R}_{t} & ={ }^{b} \boldsymbol{R}_{0}{ }^{0} \boldsymbol{R}_{1}{ }^{1} \boldsymbol{R}_{2}{ }^{2} \boldsymbol{R}_{3}{ }^{3} \boldsymbol{R}_{4}{ }^{4} \boldsymbol{R}_{5}{ }^{5} \boldsymbol{R}_{t}
\end{aligned}
$$

where

$$
\begin{aligned}
{ }^{0} \boldsymbol{R}_{m} & =\prod_{i=0}^{m-1}{ }^{i} \boldsymbol{R}_{i+1} \\
{ }^{1} \boldsymbol{p}_{w s} & =\left[\begin{array}{lll}
l_{h} & 0 & -l_{w}
\end{array}\right]^{T} \\
{ }^{4} \boldsymbol{p}_{s e} & =\left[\begin{array}{lll}
0 & -l_{b} & 0
\end{array}\right]^{T} \\
{ }^{5} \boldsymbol{p}_{e t} & =\left[\begin{array}{lll}
0 & 0 & -l_{f}
\end{array}\right]^{T} .
\end{aligned}
$$

\section{Inverse Kinematics}

Substituting (16) and (17) into (9), we have

$$
\begin{aligned}
{ }^{b} \boldsymbol{p}_{t}^{d} & ={ }^{b} \boldsymbol{R}_{0}\left({ }^{0} \boldsymbol{R}_{1}{ }^{1} \boldsymbol{p}_{w s}+{ }^{0} \boldsymbol{R}_{4}{ }^{4} \boldsymbol{p}_{s e}+{ }^{0} \boldsymbol{R}_{5}{ }^{5} \boldsymbol{p}_{e t}\right) \\
{ }^{b} \boldsymbol{R}_{t}^{d} & ={ }^{b} \boldsymbol{R}_{n}{ }^{b} \boldsymbol{R}_{0}{ }^{0} \boldsymbol{R}_{1}{ }^{1} \boldsymbol{R}_{2}{ }^{2} \boldsymbol{R}_{3}{ }^{3} \boldsymbol{R}_{4}{ }^{4} \boldsymbol{R}_{5}{ }^{5} \boldsymbol{R}_{t} .
\end{aligned}
$$

As mentioned in Section 2.1, the consecutive three joints 2, 3, and 4 are kinematically equivalent to a spherical joint. Since any orientation can be achieved with the virtual spherical joint, the inverse kinematic problem can be decoupled into two subproblems: ${ }^{22}$ inverse position and inverse orientation problems.

Let us formulate the two subproblems. The rotation matrices in (23) involving $\theta_{1}, \theta_{5}$, and $\phi$ are moved to the left-hand side of the equation to yield

$$
{ }^{0} \boldsymbol{R}_{1}^{T{ }^{b}} \boldsymbol{R}_{0}^{T b}{ }^{b} \boldsymbol{R}_{n}^{T b}{ }^{b}{ }_{5}^{d ~}{ }^{4} \boldsymbol{R}_{5}^{T}={ }^{1} \boldsymbol{R}_{2}{ }^{2} \boldsymbol{R}_{3}{ }^{3} \boldsymbol{R}_{4}
$$

where ${ }^{b} \boldsymbol{R}_{5}^{d}={ }^{b} \boldsymbol{R}_{t}^{d}{ }^{5} \boldsymbol{R}_{t}^{T}$, which is a known matrix. Substituting (24) into (22), we have

$$
{ }^{b} \boldsymbol{p}_{t}^{d}={ }^{b} \boldsymbol{R}_{0}{ }^{0} \boldsymbol{R}_{1}{ }^{1} \boldsymbol{p}_{\mathrm{ws}}+{ }^{b} \boldsymbol{R}_{n}^{T}{ }^{b} \boldsymbol{R}_{5}^{d}\left({ }^{4} \boldsymbol{R}_{5}^{T}{ }^{4} \boldsymbol{p}_{\mathrm{se}}+{ }^{5} \boldsymbol{p}_{e t}\right) .
$$

Equation (25) represents the tip position involving three unknown variables $\theta_{1}, \theta_{5}$, and $\phi$. Hence, given the target tip position, the unknown variables are determined. Thus, the inverse position problem is to derive $\theta_{1}, \theta_{5}$, and $\phi$ to ensure (25).

Substituting the derived $\theta_{1}, \theta_{5}$, and $\phi$ into (24), all the elements in the left-hand side are known. The unknown variables in (24) are then $\theta_{2}, \theta_{3}$, and $\theta_{4}$ only. Thus, the inverse orientation problem is to derive $\theta_{2}, \theta_{3}$, and $\theta_{4}$ to satisfy (24).

In this section, how to solve the inverse position problem is discussed first. Then, the inverse orientation problem is solved. Lastly, the number of inverse kinematic solutions is investigated.

4.1. Solving inverse position problem

Rearranging the terms in (25) yields

$$
{ }^{b} \boldsymbol{p}_{t}^{d}-{ }^{b} \boldsymbol{R}_{0}{ }^{0} \boldsymbol{R}_{1}{ }^{1} \boldsymbol{p}_{w s}={ }^{b} \boldsymbol{R}_{n}^{T}{ }^{b} \boldsymbol{R}_{5}^{d}\left({ }^{4} \boldsymbol{R}_{5}^{T 4} \boldsymbol{p}_{s e}+{ }^{5} \boldsymbol{p}_{e t}\right) .
$$


Computing the square norms on both sides of (26), we obtain

$$
\begin{aligned}
& \left|{ }^{b} \boldsymbol{p}_{t}^{d}\right|^{2}+\left|{ }^{1} \boldsymbol{p}_{w s}\right|^{2}-2\left({ }^{b} \boldsymbol{p}_{t}^{d T{ }^{b}} \boldsymbol{R}_{0}{ }^{0} \boldsymbol{R}_{1}{ }^{1} \boldsymbol{p}_{w s}\right) \\
& =\left|{ }^{4} \boldsymbol{p}_{s e}\right|^{2}+\left|{ }^{5} \boldsymbol{p}_{e t}\right|^{2}+2\left({ }^{5} \boldsymbol{p}_{e t}^{T}{ }^{4} \boldsymbol{R}_{5}^{T}{ }^{T} \boldsymbol{p}_{s e}\right) .
\end{aligned}
$$

Also, premultiplying both sides of (26) by ${ }^{b} \boldsymbol{n}^{T}$, we get

$$
{ }^{b} \boldsymbol{n}^{T}{ }^{b} \boldsymbol{p}_{t}^{d}-{ }^{b} \boldsymbol{n}^{T}{ }^{b} \boldsymbol{R}_{0}{ }^{0} \boldsymbol{R}_{1}{ }^{1} \boldsymbol{p}_{w s}={ }^{b} \boldsymbol{n}^{T}{ }^{b} \boldsymbol{R}_{5}^{d}\left({ }^{4} \boldsymbol{R}_{5}^{T}{ }^{4} \boldsymbol{p}_{s e}+{ }^{5} \boldsymbol{p}_{e t}\right)
$$

The equations (27) and (28) do not include the unknown variable $\phi$. They depend only on the joint variables $\theta_{1}$ and $\theta_{5}$. Thus, we begin by deriving $\theta_{1}$ and $\theta_{5}$ from (27) and (28). Then, we derive $\phi$ from (26) using the derived $\theta_{1}$ and $\theta_{5}$.

4.1.1. Derivation of $\theta_{1}$ and $\theta_{5}$. Substituting the D-H parameters given in Table I into (27) and (28), we can transform (27) and (28) into the vector form

$$
A q_{1}+B q_{5}=c
$$

where $\boldsymbol{q}_{i}=\left[\begin{array}{ll}\cos \theta_{i} & \sin \theta_{i}\end{array}\right]^{T} \in \mathfrak{R}^{2}$, and $\boldsymbol{A} \in \mathfrak{R}^{2 \times 2}, \boldsymbol{B} \in \mathfrak{R}^{2 \times 2}$ and $\boldsymbol{c} \in \mathfrak{R}^{2}$ are given by

$$
\begin{aligned}
\boldsymbol{A} & =l_{h}\left[\begin{array}{cc}
p_{z} & p_{x} \\
n_{z} & n_{x}
\end{array}\right] \\
\boldsymbol{B} & =l_{b}\left[\begin{array}{cc}
l_{f} & 0 \\
-r_{z} & r_{x}
\end{array}\right] \\
\boldsymbol{c} & =\left[\begin{array}{c}
\frac{1}{2}\left\{p_{x}^{2}+\left(p_{y}+l_{w}\right)^{2}+p_{z}^{2}+l_{h}^{2}-l_{b}^{2}-l_{f}^{2}\right\} \\
l_{f} r_{z}+n_{x} p_{x}+n_{y}\left(p_{y}+l_{w}\right)+n_{z} p_{z}
\end{array}\right]
\end{aligned}
$$

where

$$
\begin{aligned}
{ }^{b} \boldsymbol{p}_{t}^{d} & =\left[\begin{array}{lll}
p_{x} & p_{y} & p_{z}
\end{array}\right]^{T} \\
{ }^{b} \boldsymbol{R}_{5}^{d T}{ }^{b} \boldsymbol{n} & =\left[\begin{array}{lll}
r_{x} & r_{y} & r_{z}
\end{array}\right]^{T}
\end{aligned}
$$

Thus, we have to solve the vector Eq. (29) in order to determine $\theta_{1}$ and $\theta_{5}$.

How to solve (29) depends on the ranks of the matrices $\boldsymbol{A}$ and $\boldsymbol{B}$. Since $l_{b}>0$ and $l_{f}>0$, the rank of $\boldsymbol{B}$ is 1 or 2 . On the other hand, the rank of $\boldsymbol{A}$ is either 0,1 , or 2 . However, if rank $\boldsymbol{A}=0$, i.e. $\boldsymbol{A}=\mathbf{0}, \theta_{1}$ is indeterminate. Hence this is a singular case. For now, consider nonsingular cases, because singular cases are analyzed later. As a result, four combinations of $\operatorname{rank} \boldsymbol{A}$ and $\operatorname{rank} \boldsymbol{B}$ are possible:

$$
\{\operatorname{rank} \boldsymbol{A}, \operatorname{rank} \boldsymbol{B}\}=\{\{1,1\},\{1,2\},\{2,1\},\{2,2\}\} .
$$

In the following, (29) is solved for each case.

(a) $\operatorname{rank} \boldsymbol{A}=1$ and $\operatorname{rank} \boldsymbol{B}=1$ : Let $\boldsymbol{N}_{A} \in \mathfrak{R}^{2 \times 2}$ and $\boldsymbol{N}_{B} \in \mathfrak{R}^{2 \times 2}$ be the adjoint matrices of $\boldsymbol{A}$ and $\boldsymbol{B}$, respectively. Premultiplying both sides of (29) by $N_{B}$ yields

$$
N_{B} A q_{1}+(\operatorname{det} B) q_{5}=N_{B} c .
$$

Similarly, premultiplication by $\boldsymbol{N}_{B}$ provides

$$
(\operatorname{det} \boldsymbol{A}) \boldsymbol{q}_{1}+\boldsymbol{N}_{A} \boldsymbol{B} \boldsymbol{q}_{5}=\boldsymbol{N}_{A} \boldsymbol{c} .
$$


Since $\operatorname{det} \boldsymbol{A}=0$ and $\operatorname{det} \boldsymbol{B}=0$, (33) and (34) are reduced to

$$
\begin{aligned}
& N_{B} A q_{1}=N_{B} c \\
& N_{A} B q_{5}=N_{A} c .
\end{aligned}
$$

It can be verified that $N_{B} A$ is the adjoint matrix of $N_{A} B$, and vice versa. Therefore, rank $N_{B} A=\operatorname{rank} N_{A} B$. Since the ranks of $A$ and $B$ are one, the ranks of $N_{B} A$ and $N_{A} B$ are one or zero. If rank $\boldsymbol{N}_{B} \boldsymbol{A}=\operatorname{rank} \boldsymbol{N}_{A} \boldsymbol{B}=0, \theta_{1}$, and $\theta_{5}$ are indeterminate, hence this is a singular case. This singular case is analyzed later.

Suppose that rank $N_{B} A=\operatorname{rank} N_{A} B=1$. Let $u \in \Re^{2}$ and $v \in \mathfrak{R}^{2}$ be nonzero basis vectors of $N_{B} \boldsymbol{A}$ and $\boldsymbol{N}_{A} \boldsymbol{B}$ respectively. Because $\operatorname{det} \boldsymbol{B}=l_{b}^{2} l_{f} r_{x}=0$, it is obvious that $r_{x}=0$. Also, since $N_{B} A \neq \mathbf{0}$, it is guaranteed that

$$
\left(r_{z} p_{x}+l_{f} n_{x}\right)^{2}+\left(r_{z} p_{z}+l_{f} n_{z}\right)^{2}>0
$$

Therefore, $\boldsymbol{u}$ and $\boldsymbol{v}$ can be chosen so that

$$
\begin{aligned}
\boldsymbol{u} & =\left[\begin{array}{ll}
0 & 1
\end{array}\right]^{T} \\
\boldsymbol{v} & =\left[\begin{array}{ll}
r_{z} p_{x}+l_{f} n_{x} & -r_{z} p_{z}-l_{f} n_{z}
\end{array}\right]^{T} .
\end{aligned}
$$

Premultiplying both sides of (35) by $\boldsymbol{u}^{T}$, we obtain

$$
\boldsymbol{u}^{T} \boldsymbol{N}_{B} \boldsymbol{A} \boldsymbol{q}_{1}=\boldsymbol{u}^{T} \boldsymbol{N}_{B} \boldsymbol{c}
$$

Similarly, premultiplying both sides of (36) by $\boldsymbol{v}^{T}$, we have

$$
\boldsymbol{v}^{T} \boldsymbol{N}_{A} \boldsymbol{B} \boldsymbol{q}_{5}=\boldsymbol{v}^{T} \boldsymbol{N}_{A} \boldsymbol{c}
$$

From (40), $\boldsymbol{q}_{1}$ is derived (see Appendix A):

$$
\boldsymbol{q}_{1}=\frac{1}{\boldsymbol{x}_{u}^{T} \boldsymbol{x}_{u}}\left[y_{u} \boldsymbol{I}_{2} \pm \sqrt{\boldsymbol{x}_{u}^{T} \boldsymbol{x}_{u}-y_{u}^{2}} \cdot \boldsymbol{H}\right] \boldsymbol{x}_{u}
$$

where

$$
\begin{aligned}
\boldsymbol{x}_{u} & =\boldsymbol{A}^{T} \boldsymbol{N}_{B}^{T} \boldsymbol{u} \\
y_{u} & =\boldsymbol{u}^{T} \boldsymbol{N}_{B} \boldsymbol{c} .
\end{aligned}
$$

In a similar way, $\boldsymbol{q}_{5}$ is derived from (41):

$$
\boldsymbol{q}_{5}=\frac{1}{\boldsymbol{x}_{v}^{T} \boldsymbol{x}_{v}}\left[y_{v} \boldsymbol{I}_{2} \pm \sqrt{\boldsymbol{x}_{v}^{T} \boldsymbol{x}_{v}-y_{v}^{2}} \cdot \boldsymbol{H}\right] \boldsymbol{x}_{v}
$$

where

$$
\begin{aligned}
\boldsymbol{x}_{v} & =\boldsymbol{B}^{T} \boldsymbol{N}_{A}^{T} \boldsymbol{v} \\
y_{v} & =\boldsymbol{v}^{T} \boldsymbol{N}_{A} \boldsymbol{c} .
\end{aligned}
$$

Thus, using the arctangent function, we can compute $\theta_{1}$ and $\theta_{5}$ from (42) and (43). 
(b) rank $\boldsymbol{A}=1$ and rank $\boldsymbol{B}=2$ : Since $\operatorname{det} \boldsymbol{A}=0$, we can obtain $\theta_{5}$ from (43) as analogous to the case (a). However, since $r_{x} \neq 0$, there is a special case that (37) does not hold. In such a case, $\boldsymbol{v}$ given by (39) becomes zero vector. Hence, if (37) is not ensured, $\boldsymbol{v}$ has to be replaced with

$$
\boldsymbol{v}=\left[\begin{array}{ll}
-p_{x} & p_{z}
\end{array}\right]^{T} .
$$

It can be verified that this is a nonzero vector, because the condition

$$
p_{x}^{2}+p_{z}^{2}>0
$$

must be satisfied if (37) is not ensured.

From (33),

$$
\boldsymbol{u}^{T} \boldsymbol{N}_{B} \boldsymbol{A} \boldsymbol{q}_{1}=\boldsymbol{u}^{T} \boldsymbol{N}_{B} \boldsymbol{c}-(\operatorname{det} B) \boldsymbol{u}^{T} \boldsymbol{q}_{5}
$$

where $\boldsymbol{u}$ is given by (38) if (37) is satisfied. If not, $\boldsymbol{u}$ has to be replaced with

$$
\boldsymbol{u}=\left[\begin{array}{ll}
1 & 0
\end{array}\right]^{T} .
$$

Substituting $\boldsymbol{q}_{5}$ given by (43) into (46), $\theta_{1}$ can be obtained.

Note that the ranks of $\boldsymbol{N}_{A} B$ and $N_{B} A$ are always one, because $B$ is of full rank. Hence, no singularity occurs in this case.

(c) $\operatorname{rank} \boldsymbol{A}=2$ and $\operatorname{rank} \boldsymbol{B}=1$ : Since $\operatorname{det} \boldsymbol{B}=0, \theta_{1}$ can be obtained from (42), where $\boldsymbol{u}$ is given by (38) if (37) holds, otherwise by (47).

From (34),

$$
\boldsymbol{v}^{T} \boldsymbol{N}_{A} \boldsymbol{B} \boldsymbol{q}_{5}=\boldsymbol{v}^{T} \boldsymbol{N}_{A} \boldsymbol{c}-(\operatorname{det} A) \boldsymbol{v}^{T} \boldsymbol{q}_{1}
$$

where $\boldsymbol{v}$ is given by (39) if (37) holds, otherwise by (44). Substituting $\boldsymbol{q}_{1}$ given by (42) into (48), $\theta_{5}$ can be obtained.

(d) rank $\boldsymbol{A}=2$ and rank $\boldsymbol{B}=2$ : Since $\boldsymbol{B}$ is invertible, (29) can be transformed into

$$
\boldsymbol{q}_{5}=-\boldsymbol{B}^{-1}\left(A \boldsymbol{q}_{1}-\boldsymbol{c}\right) .
$$

Since $\boldsymbol{q}_{5}$ is a unit vector, the constraint condition

$$
\boldsymbol{q}_{5}^{T} \boldsymbol{q}_{5}-1=0
$$

must be satisfied. Substituting (49) into (50), we obtain the equation involving only $\theta_{1}$ as

$$
\boldsymbol{q}_{1}^{T} \boldsymbol{W}^{T} \boldsymbol{W} \boldsymbol{q}_{1}-2 \boldsymbol{c}^{T} \boldsymbol{B}^{-T} \boldsymbol{W} \boldsymbol{q}_{1}+\boldsymbol{c}^{T} \boldsymbol{B}^{-T} \boldsymbol{B}^{-1} \boldsymbol{c}-1=0
$$

where $\boldsymbol{W}=\boldsymbol{B}^{-1} \boldsymbol{A}$. Thus, $\theta_{1}$ can be obtained by solving (51). It can be verified that (51) is transformed into a quartic equation by using the half angle formula. Although a quartic equation is analytically solvable, the actual computation is an onerous work. For this reason, a smart computation method is developed in the following.

Let $\lambda_{1}$ and $\lambda_{2}$ be eigenvalues of the matrix $\boldsymbol{W}^{T} \boldsymbol{W}$. Since $\boldsymbol{W}^{T} \boldsymbol{W}$ is positive definite, $\lambda_{1}$ and $\lambda_{2}$ are real positive values. Without loss of generality, $\lambda_{1} \leq \lambda_{2}$. Further, let $\boldsymbol{u}_{1}$ and $\boldsymbol{u}_{2}$ be unit eigenvectors associated with $\lambda_{1}$ and $\lambda_{2}$, respectively. Then, since $\boldsymbol{u}_{1}$ and $\boldsymbol{u}_{2}$ are orthogonal to each other,

$$
\boldsymbol{W}^{T} \boldsymbol{W}=\boldsymbol{U} \boldsymbol{\Lambda} \boldsymbol{U}^{T}=\lambda_{1}\left[\boldsymbol{u}_{1} \boldsymbol{u}_{1}^{T}\right]+\lambda_{2}\left[\boldsymbol{u}_{2} \boldsymbol{u}_{2}^{T}\right]
$$

where $\boldsymbol{U}=\left[\begin{array}{ll}\boldsymbol{u}_{1} & \boldsymbol{u}_{2}\end{array}\right]$ and $\boldsymbol{\Lambda}=\operatorname{diag}\left\{\lambda_{1}, \lambda_{2}\right\}$. How to compute the eigenvalues and eigenvectors is presented in Appendix B. In addition, let us define a new vector $\boldsymbol{k} \in \mathfrak{R}^{2}$ described by

$$
\boldsymbol{k}=\left[\begin{array}{ll}
k_{1} & k_{2}
\end{array}\right]^{T}=\boldsymbol{U}^{T} \boldsymbol{W}^{T} \boldsymbol{B}^{-1} \boldsymbol{c} .
$$


Substituting (52) and (53) into (51), we have

$$
\boldsymbol{q}_{1}^{T} \boldsymbol{U} \boldsymbol{\Lambda} \boldsymbol{U}^{T} \boldsymbol{q}_{1}-2 \boldsymbol{k}^{T} \boldsymbol{U}^{T} \boldsymbol{q}_{1}+\boldsymbol{k}^{T} \boldsymbol{\Lambda}^{-1} \boldsymbol{k}-1=0 .
$$

Since $\boldsymbol{U}$ is orthonormal, there exists $\psi \in \Re$ which satisfies

$$
\left[\begin{array}{c}
\cos \psi \\
\sin \psi
\end{array}\right]=\boldsymbol{U}^{T} \boldsymbol{q}_{1}
$$

Substituting (55) into (54) yields

$$
\lambda_{1} C_{\psi}^{2}+\lambda_{2} S_{\psi}^{2}-2 k_{1} C_{\psi}-2 k_{2} S_{\psi}+\frac{k_{1}^{2}}{\lambda_{1}}+\frac{k_{2}^{2}}{\lambda_{2}}-1=0
$$

where $C_{\psi}=\cos \psi$ and $S_{\psi}=\sin \psi$. If $k_{1}=0$, (56) can be transformed into a quadratic equation in terms of $S_{\psi}$ :

$$
\left(\lambda_{2}-\lambda_{1}\right) S_{\psi}^{2}-2 k_{2} S_{\psi}+\lambda_{1}+\frac{k_{2}^{2}}{\lambda_{2}}-1=0 .
$$

Similarly, if $k_{2}=0,(56)$ is written by a quadratic equation in terms of $C_{\psi}$ :

$$
\left(\lambda_{1}-\lambda_{2}\right) C_{\psi}^{2}-2 k_{1} C_{\psi}+\lambda_{2}+\frac{k_{1}^{2}}{\lambda_{1}}-1=0
$$

Also, if $\lambda_{1}=\lambda_{2} \equiv \lambda$, (56) reduces to a first-order trigonometric equation in terms of $\psi$ :

$$
2 k_{1} C_{\psi}+2 k_{2} S_{\psi}-\lambda-\frac{k_{1}^{2}+k_{2}^{2}}{\lambda}+1=0 .
$$

Hence, if $k_{1}=0$ or $k_{2}=0$, or $\lambda_{1}=\lambda_{2}, \psi$ is readily derived. $\theta_{1}$ is then obtained from (55).

Consider a general case that $k_{1} \neq 0, k_{2} \neq 0$ and $\lambda_{1} \neq \lambda_{2}$. Since

$$
e-e\left(C_{\psi}^{2}+S_{\psi}^{2}\right)=0
$$

for any scalar $e \in \Re$, adding (57) to (56), we finally acquire

$$
\left(\lambda_{1}-e\right)\left(C_{\psi}-\frac{k_{1}}{\lambda_{1}-e}\right)^{2}+\left(\lambda_{2}-e\right)\left(S_{\psi}-\frac{k_{2}}{\lambda_{2}-e}\right)^{2}+f(e)=0
$$

where

$$
f(e)=e-\frac{k_{1}^{2}}{\lambda_{1}-e}-\frac{k_{2}^{2}}{\lambda_{2}-e}+\frac{k_{1}^{2}}{\lambda_{1}}+\frac{k_{2}^{2}}{\lambda_{2}}-1 .
$$

Now, let us compute $e_{0}$ so that $f\left(e_{0}\right)=0$. Obviously, $f(e)$ is indeterminate at $e=\lambda_{1}$ and $e=\lambda_{2}$. Computing the right-hand and left-hand limits around $\lambda_{1}$ and $\lambda_{2}$, respectively, we have

$$
\begin{aligned}
& \lim _{e \rightarrow \lambda_{1}+0} f(e)=+\infty \\
& \lim _{e \rightarrow \lambda_{2}-0} f(e)=-\infty .
\end{aligned}
$$

According to the intermediate value theorem, these results guarantee that there exists at least one real solution $e_{0}$ between $\lambda_{1}$ and $\lambda_{2}$. Thus, we can always find $e_{0}$ which satisfies that $\lambda_{1}<e_{0}<\lambda_{2}$. Within 
this range, $f\left(e_{0}\right)=0$ can be rewritten by

$$
e_{0}^{3}+\left\{\frac{k_{1}^{2}}{\lambda_{1}}+\frac{k_{2}^{2}}{\lambda_{2}}-\left(\lambda_{1}+\lambda_{2}\right)-1\right\} e_{0}^{2}-\left\{\frac{\lambda_{2}}{\lambda_{1}} k_{1}^{2}+\frac{\lambda_{1}}{\lambda_{2}} k_{2}^{2}-\left(\lambda_{1}+\lambda_{2}\right)-\lambda_{1} \lambda_{2}\right\} e_{0}-\lambda_{1} \lambda_{2}=0 .
$$

Thus, we have to solve the cubic Eq. (60) to get $e_{0}$. Solving a cubic equation is relatively easy in comparison with solving a quartic equation. There are several analytical methods of solving a cubic equation, for example, Cardano's method. ${ }^{24}$ Due to the limited space of this paper, the derivation of $e_{0}$ is omitted.

Substituting the solution $e_{0}$ into (58), we have

$$
\left(\lambda_{1}-e_{0}\right)\left(C_{\psi}-\frac{k_{1}}{\lambda_{1}-e_{0}}\right)^{2}+\left(\lambda_{2}-e_{0}\right)\left(S_{\psi}-\frac{k_{2}}{\lambda_{2}-e_{0}}\right)^{2}=0 .
$$

Since $\lambda_{1}-e_{0}<0$ and $\lambda_{2}-e_{0}>0$, we can factorize (61) into two equations

$$
\begin{aligned}
& \boldsymbol{s}_{1}^{T} \boldsymbol{U}^{T} \boldsymbol{q}_{1}=2 \frac{\boldsymbol{s}_{1}^{T} \boldsymbol{H} \boldsymbol{k}}{\boldsymbol{s}_{2}^{T} \boldsymbol{H} \boldsymbol{s}_{1}} \\
& \boldsymbol{s}_{2}^{T} \boldsymbol{U}^{T} \boldsymbol{q}_{1}=2 \frac{\boldsymbol{s}_{2}^{T} \boldsymbol{H} \boldsymbol{k}}{\boldsymbol{s}_{1}^{T} \boldsymbol{H} \boldsymbol{s}_{2}}
\end{aligned}
$$

where $\boldsymbol{H}$ is given by (93), and $s_{1} \in \mathfrak{R}^{2}$ and $\boldsymbol{s}_{2} \in \mathfrak{R}^{2}$ are given by

$$
\begin{aligned}
& \boldsymbol{s}_{1}=\left[\begin{array}{ll}
\sqrt{e_{0}-\lambda_{1}} & \sqrt{\lambda_{2}-e_{0}}
\end{array}\right]^{T} \\
& \boldsymbol{s}_{2}=\left[\begin{array}{ll}
\sqrt{e_{0}-\lambda_{1}} & -\sqrt{\lambda_{2}-e_{0}}
\end{array}\right]^{T} .
\end{aligned}
$$

To ensure (61), either (62) or (63) has to be satisfied. Since (62) and (63) are first-order trigonometric equations, $\boldsymbol{q}_{1}$ can be derived as shown in Appendix A. Substituting the derived $\boldsymbol{q}_{1}$ into (49), $\boldsymbol{q}_{5}$ is readily derived. Using the arctangent function, we can finally obtain $\theta_{1}$ and $\theta_{5}$.

4.1.2. Derivation of $\phi$. Substituting (10) into (26), we have

$$
\boldsymbol{y}=\left\{\cos \phi \boldsymbol{I}-\sin \phi\left[{ }^{b} \boldsymbol{n} \times\right]+(1-\cos \phi)\left[{ }^{b} \boldsymbol{n}^{b} \boldsymbol{n}^{T}\right]\right\} \boldsymbol{x}
$$

where

$$
\begin{aligned}
& \boldsymbol{y}={ }^{b} \boldsymbol{p}_{t}^{d}-{ }^{b} \boldsymbol{R}_{0}{ }^{0} \boldsymbol{R}_{1}{ }^{1} \boldsymbol{p}_{w s} \\
& \boldsymbol{x}={ }^{b} \boldsymbol{R}_{5}^{d}\left({ }^{4} \boldsymbol{R}_{5}^{T 4} \boldsymbol{p}_{s e}+{ }^{5} \boldsymbol{p}_{e t}\right) .
\end{aligned}
$$

Since $\theta_{1}$ and $\theta_{5}$ have been determined, $\boldsymbol{y}$ and $\boldsymbol{x}$ are known vectors.

Computing the cross product with ${ }^{b} \boldsymbol{n}$ on both sides of (66), we obtain

$$
{ }^{b} \boldsymbol{n} \times \boldsymbol{y}=\cos \phi\left({ }^{b} \times \boldsymbol{x}\right)-\sin \phi\left\{{ }^{b} \boldsymbol{n} \times\left({ }^{b} \boldsymbol{n} \times \boldsymbol{x}\right)\right\} .
$$

From this, if ${ }^{b} \boldsymbol{n} \times \boldsymbol{x} \neq \mathbf{0}$, we can derive $\cos \phi$ and $\sin \phi$ :

$$
\begin{aligned}
& \cos \phi=\frac{\left({ }^{b} \boldsymbol{n} \times \boldsymbol{x}\right)^{T}\left({ }^{b} \boldsymbol{n} \times \boldsymbol{y}\right)}{\left({ }^{b} \boldsymbol{n} \times \boldsymbol{x}\right)^{T}\left({ }^{b} \boldsymbol{n} \times \boldsymbol{x}\right)} \\
& \sin \phi=\frac{\boldsymbol{x}^{T}\left({ }^{b} \boldsymbol{n} \times \boldsymbol{y}\right)}{\boldsymbol{x}^{T} \boldsymbol{x}-\left({ }^{b} \boldsymbol{n}^{T} \boldsymbol{x}\right)^{2}}
\end{aligned}
$$


Thus, $\phi$ is uniquely determined from (69) and (70). Note that, if ${ }^{b} \boldsymbol{n} \times \boldsymbol{x}=\mathbf{0}, \phi$ is indeterminate, hence this is a singular case. How to deal with this singular case is discussed later.

\subsection{Solving inverse orientation problem}

To this point, the inverse position problem has been solved. Hence, $\theta_{1}, \theta_{5}$, and $\phi$ are known. Substitution of them into (24) makes the left-hand side of the equation known. The right-hand side is described by

$$
\begin{aligned}
{ }^{1} \boldsymbol{R}_{2}{ }^{2} \boldsymbol{R}_{3}{ }^{3} \boldsymbol{R}_{4} & \\
= & {\left[\begin{array}{ccc}
* & \cos \theta_{2} \cos \theta_{3} & * \\
* & \sin \theta_{2} \cos \theta_{3} & * \\
-\cos \theta_{3} \sin \theta_{4} & -\sin \theta_{3} & \cos \theta_{3} \cos \theta_{4}
\end{array}\right] }
\end{aligned}
$$

where the elements denoted by $*$ are omitted. Combining some elements, we can derive

$$
\begin{aligned}
& \boldsymbol{q}_{2}= \pm \frac{1}{\sqrt{R_{12}^{2}+R_{22}^{2}}}\left[\begin{array}{l}
R_{12} \\
R_{22}
\end{array}\right] \\
& \boldsymbol{q}_{3}=\left[\begin{array}{c} 
\pm \sqrt{1-R_{32}^{2}} \\
-R_{32}
\end{array}\right] \\
& \boldsymbol{q}_{4}= \pm \frac{1}{\sqrt{R_{31}^{2}+R_{33}^{2}}}\left[\begin{array}{c}
R_{33} \\
-R_{31}
\end{array}\right]
\end{aligned}
$$

where $R_{i j}$ stands for the $(i, j)$ element of the rotation matrix on the left-hand side of (24), and all double signs correspond to each other. Using the arctangent function, we can obtain $\theta_{2}, \theta_{3}$, and $\theta_{4}$ from (71), (72), and (73), respectively.

It should be noted that two different sets of solutions $\theta_{2}, \theta_{3}$, and $\theta_{4}$ are always possible for nonsingular cases. As inferred from (71) and (73), there is a singular case that $\theta_{2}$ and $\theta_{4}$ are indeterminate. This singular case is analyzed later.

\subsection{Number of solutions}

Since the manipulator considered in this paper has a special geometry, the inverse kinematic solution is not unique. ${ }^{10}$ Let us evaluate the maximum number of solutions.

The first step of the inverse kinematic computation is to determine $\theta_{1}$ and $\theta_{5}$ so that (29) is ensured. As described in Section 4.1.1, (29) is decomposed into two first-order trigonometric equations, and each trigonometric equation has at most two different solutions. Therefore, the maximum number of solution sets of $\theta_{1}$ and $\theta_{5}$ is four.

The second step is to compute $\phi$ for each set of $\theta_{1}$ and $\theta_{5}$. Since $\phi$ is determined from $\cos \phi$ and $\sin \phi$ given by (69) and (70) respectively, $\phi$ is unique for each set of $\theta_{1}$ and $\theta_{5}$.

The last step is to determine $\theta_{2}, \theta_{3}$, and $\theta_{4}$. As shown in (71), (72), and (73), two different solution sets are possible for each set of $\theta_{1}, \theta_{5}$, and $\phi$.

Consequently, the maximum number of solution sets is given by $4 \times 1 \times 2=8$. Note that selection of a solution set is arbitrary from the mathematical perspective. The operator has to choose an appropriate solution set by considering various physical constraints such as joint limits and the movable space of the manipulator.

\section{Singularity Analysis}

In general, there are two types of singularities for a serial manipulator, as shown in ref. [25]. One is the saturation singularity, and the other is the internal singularity. The saturation singularity occurs when the target tip position is on or outside the boundary of the manipulator's workspace. The internal singularity occurs inside the workspace. In this section, the conditions for the saturation and internal singularities are derived. 


\subsection{Saturation singularity}

We may encounter the saturation singularity when solving the inverse position problem. As described in Section 4.1.1, the vector equation (29) is decomposed into two first-order trigonometric equations, for example, (35) and (36). Each equation is of the form

$$
\boldsymbol{x}_{i}^{T} \boldsymbol{q}_{i}=y_{i}, \quad i=1 \text { or } 5
$$

where $\boldsymbol{x}_{i} \in \mathfrak{R}^{2}$ and $y_{i}$ are constant vector and scalar respectively. If $\left|\boldsymbol{x}_{i}\right|$ is less than $y_{i}$, no real solutions exist. Therefore, the condition for the saturation singularity is described by

$$
\boldsymbol{x}_{i}^{T} \boldsymbol{x}_{i}<y_{i}^{2}
$$

\subsection{Internal singularity}

Identification of all internal singular configurations is especially important, because we have to carefully avoid these singular configurations whenever we operate the manipulator. If the manipulator is at a singular configuration, the inverse position or orientation problem is unsolvable. In the following, we first derive the singular conditions at which the inverse position problem is unsolvable. Then, we derive the singular conditions associated with the inverse orientation problem.

5.2.1. Singular configurations at which inverse position problem is unsolvable. As described in Section 4.1, $\theta_{1}$ and $\theta_{5}$ are determined so that (29) is satisfied, and $\phi$ is determined by solving (66). We may encounter two singular cases in solving (29) and one singular case in solving (66).

The first singular case occurs when the rank of the matrix $\boldsymbol{A}$ given by (30) is zero. Namely, the conditions for this singular case is given by

$$
\boldsymbol{A}=l_{h}\left[\begin{array}{ll}
p_{z} & p_{x} \\
n_{z} & n_{x}
\end{array}\right]=\left[\begin{array}{ll}
0 & 0 \\
0 & 0
\end{array}\right]
$$

In this case, $\theta_{1}$ is indeterminate, because $\boldsymbol{q}_{1}$ vanishes from (29). Since $p_{x}=0$ and $p_{z}=0$, the target tip position is on the axis of joint 1 . Furthermore, since $n_{x}=0$ and $n_{z}=0$, the unconstrained axis is parallel to the joint axis 1 .

The second singular case arises when both the matrices $N_{B} A$ and $N_{A} B$ are zero matrices. In this case, both $\theta_{1}$ and $\theta_{5}$ are indeterminate. From $\boldsymbol{N}_{B} \boldsymbol{A}=\mathbf{0}$ or $\boldsymbol{N}_{A} \boldsymbol{B}=\mathbf{0}$, we can derive the conditions for this singular case:

$$
\begin{aligned}
r_{x} & =0 \\
r_{z} p_{x}+l_{f} n_{x} & =0 \\
r_{z} p_{z}+l_{f} n_{z} & =0 .
\end{aligned}
$$

The third singular case occurs when $\boldsymbol{x}$ given by (68) is parallel to the unconstrained axis defined by ${ }^{b} \boldsymbol{n}$. In this case, $\phi$ is indeterminate, because (66) reduces to

$$
\begin{aligned}
\boldsymbol{y} & =\left\{\cos \phi \boldsymbol{I}-\sin \phi\left[{ }^{b} \boldsymbol{n} \times\right]+(1-\cos \phi)\left[{ }^{b} \boldsymbol{n}^{b} \boldsymbol{n}^{T}\right]\right\} \boldsymbol{x} \\
& =\boldsymbol{x} .
\end{aligned}
$$

This yields the conditions for this singular case:

$$
\begin{aligned}
& { }^{b} \boldsymbol{n} \times \boldsymbol{y}={ }^{b} \boldsymbol{n} \times{ }^{b} \boldsymbol{p}_{t}^{d}-{ }^{b} \boldsymbol{n} \times{ }^{b} \boldsymbol{R}_{0}{ }^{0} \boldsymbol{R}_{1}{ }^{1} \boldsymbol{p}_{w s}=\mathbf{0} \\
& { }^{b} \boldsymbol{n} \times \boldsymbol{x}={ }^{b} \boldsymbol{n} \times{ }^{b} \boldsymbol{R}_{5}^{d}\left({ }^{4} \boldsymbol{R}_{5}^{T 4} \boldsymbol{p}_{s e}+{ }^{5} \boldsymbol{p}_{e t}\right)=\mathbf{0} .
\end{aligned}
$$

The conditions (78) and (79) involve the joint angles $\theta_{1}$ and $\theta_{5}$ respectively. To eliminate the joint angles, (78) and (79) are solved for $\theta_{1}$ and $\theta_{5}$ respectively, and then the obtained $\theta_{1}$ and $\theta_{5}$ are 
substituted into (29). The detailed computation is omitted due to the space limit. As a result, the conditions for this singular case are obtained as follows:

- If $n_{y} \neq 0$ :

$$
\begin{aligned}
& \left\{p_{x}-\frac{n_{x}}{n_{y}}\left(p_{y}+l_{w}\right)\right\}^{2}+\left\{p_{z}-\frac{n_{z}}{n_{y}}\left(p_{y}+l_{w}\right)\right\}^{2}=l_{h}^{2} \\
& r_{y}=0 \\
& r_{z}=\frac{1}{2 l_{f}}\left\{\frac{n_{y}}{p_{y}+l_{w}}\left(l_{b}^{2}-l_{f}^{2}\right)-\frac{p_{y}+l_{w}}{n_{y}}\right\}
\end{aligned}
$$

- If $n_{y}=0$ :

$$
\begin{aligned}
& p_{y}=-l_{w} \\
& r_{y}=0 \\
& l_{b}^{2}-l_{f}^{2}+l_{f}^{2} r_{z}^{2}=\left\{\frac{L_{x}^{2}+L_{z}^{2}-l_{h}^{2}+l_{b}^{2}-l_{f}^{2}+l_{f}^{2} r_{z}^{2}}{2\left(n_{x} p_{x}+n_{z} p_{z}+l_{f} r_{z}\right)}\right\}^{2} .
\end{aligned}
$$

where $L_{x}=p_{x}+l_{f} r_{z} n_{x}$ and $L_{z}=p_{z}+l_{f} r_{z} n_{z}$.

Note that $\phi$ can be chosen arbitrarily in this singular case. This is because selection of $\phi$ does not affect the joint angles $\theta_{1}$ and $\theta_{5}$. Also, the other joint angles $\theta_{2}, \theta_{3}$, and $\theta_{4}$ always exist for any $\phi$. Therefore, this singular case is not a kinematic singularity. This is a mathematical singularity. A simple remedy for this singularity is to set $\phi$ to zero. When $\phi=0$, the achieved tip orientation is exactly same as the desired one.

5.2.2. Singular configurations at which inverse orientation problem is unsolvable. The solutions to the inverse orientation problem are given by (71), (72), and (73). If $\left|R_{32}\right|=1$, it is obvious that $R_{12}=R_{22}=0$ and $R_{31}=R_{33}=0$. In this case, $\theta_{2}$ and $\theta_{4}$ are indeterminate. From (24), the condition for this singular case is obtained as

$$
\left|\boldsymbol{e}_{3}^{T 0} \boldsymbol{R}_{1}^{T{ }^{b}} \boldsymbol{R}_{0}^{T{ }^{b}} \boldsymbol{R}_{n}^{T{ }^{b}} \boldsymbol{R}_{5}^{d{ }^{4}} \boldsymbol{R}_{5}^{T} \boldsymbol{e}_{2}\right|=1
$$

where $\boldsymbol{e}_{2}=\left[\begin{array}{lll}0 & 1 & 0\end{array}\right]^{T}$, and $\boldsymbol{e}_{3}=\left[\begin{array}{lll}0 & 0 & 1\end{array}\right]^{T}$. This condition depends on $\theta_{1}, \theta_{5}$, and $\phi$, which are determined by solving the inverse position problem. Hence, substituting them into (86), we may be able to derive the singular condition described in terms of the target tip position and orientation. However, derivation of the condition in an explicit form is extremely difficult, because $\theta_{1}$ and $\theta_{5}$ are not given in explicit forms. From the pragmatic standpoint, however, explicit formulation of the condition is not necessary. To check whether this singularity occurs or not, we have only to solve the inverse position problem for given target position and orientation, and then evaluate the condition (86).

\section{Examples}

Let us verify the validity of the inverse kinematic computation method derived in Section 4 by numerical examples. In the following examples, the link lengths are fixed to

$$
\begin{array}{ll}
l_{h}=0.07(\mathrm{~m}), & l_{w}=0.103(\mathrm{~m}), \\
l_{b}=0.13(\mathrm{~m}), & l_{f}=0.045(\mathrm{~m}) .
\end{array}
$$

In addition, the unconstrained direction vector ${ }^{b} \boldsymbol{n}$ is assumed to be

$$
{ }^{b} \boldsymbol{n}=\left[\begin{array}{lll}
0 & 0 & 1
\end{array}\right]^{T} .
$$


To check the consistency of the inverse kinematics, we begin by computing the forward kinematics. As an example, consider the following joint angles:

$$
\boldsymbol{\theta}=\left[\begin{array}{lllll}
30 & -30 & -45 & 90 & 45
\end{array}\right]^{T}(\mathrm{deg})
$$

The tip position and orientation at these joint angles are obtained from the forward kinematic Eqs. (16) and (17). The results are

$$
{ }^{b} \boldsymbol{p}_{t}=\left[\begin{array}{r}
0.035 \\
-0.240 \\
-0.031
\end{array}\right], \quad{ }^{b} \boldsymbol{R}_{t}=\left[\begin{array}{ccc}
0 & -1 & 0 \\
0 & 0 & 1 \\
-1 & 0 & 0
\end{array}\right]
$$

Next, we compute the inverse kinematics by using these tip position and orientation. Since both $\boldsymbol{A}$ and $\boldsymbol{B}$ in (29) are of full rank, $\theta_{1}$ is computed from (62) and (63). Computing the parameters in (62) and (63), we obtain

$$
\begin{aligned}
{\left[\begin{array}{ll}
\lambda_{1} & \lambda_{2}
\end{array}\right]^{T} } & =\left[\begin{array}{ll}
0.101 & 0.505
\end{array}\right]^{T} \\
{\left[\begin{array}{ll}
k_{1} & k_{2}
\end{array}\right]^{T} } & =\left[\begin{array}{ll}
0.073 & 0.424
\end{array}\right]^{T} \\
\boldsymbol{U} & =\left[\begin{array}{rr}
0.430 & -0.903 \\
0.903 & 0.430
\end{array}\right] \\
e_{0} & =0.106 .
\end{aligned}
$$

From these values, $s_{1}^{T} s_{1}=s_{2}^{T} s_{2}=0.404$, and

$$
\begin{aligned}
& \left(2 \frac{\boldsymbol{s}_{1}^{T} \boldsymbol{H} \boldsymbol{k}}{\boldsymbol{s}_{2}^{T} \boldsymbol{H} \boldsymbol{s}_{1}}\right)^{2}=0.088<\boldsymbol{s}_{1}^{T} \boldsymbol{s}_{1} \\
& \left(2 \frac{\boldsymbol{s}_{2}^{T} \boldsymbol{H} \boldsymbol{k}}{\boldsymbol{s}_{1}^{T} \boldsymbol{H} \boldsymbol{s}_{2}}\right)^{2}=2.686>\boldsymbol{s}_{2}^{T} \boldsymbol{s}_{2} .
\end{aligned}
$$

Therefore, (62) is solvable, but (63) is not. Solving (62), we get $\theta_{1}=\{30,-94.519\}$ (deg). For each $\theta_{1}, \theta_{5}$ is uniquely determined from (49): $\theta_{5}=\{45,11.441\}$ (deg). Then, for each set of $\theta_{1}$ and $\theta_{5}, \phi$ is uniquely determined from (69) and (70): $\phi=\{0,-37.425\}$ (deg). Lastly, $\theta_{2}, \theta_{3}$, and $\theta_{4}$ are computed from (71), (72), and (73) respectively. As described in Section 4.2, two different sets of $\theta_{2}, \theta_{3}$, and $\theta_{4}$ are always obtained for each set of $\theta_{1}, \theta_{5}$, and $\phi$. Consequently, we have four sets of joint angles $\boldsymbol{\theta}$ and $\phi$ :

$$
\begin{aligned}
& {[\boldsymbol{\theta} \mid \phi]^{T}(\mathrm{deg})=} \\
& \left\{\begin{array}{llllll}
{\left[\begin{array}{lllll}
30 & -30 & -45 & 90 & 45 \mid 0
\end{array}\right]^{T}} \\
{\left[\begin{array}{llllll}
30 & 150 & -135 & -90 & 45 \mid 0
\end{array}\right]^{T}} \\
{\left[\begin{array}{lllll}
-94.519 & -30 & -45 & 90 & 11.441 \mid-37.425
\end{array}\right]^{T}} \\
{\left[\begin{array}{lllll}
-94.519 & 150 & -135 & -90 & 11.441 \mid-37.425
\end{array}\right]^{T}}
\end{array}\right.
\end{aligned}
$$

As shown, the first solution set is equal to (87). Moreover, since the target tip position and orientation are reachable, the unconstrained rotation angle $\phi$ is zero. These results are consistent with the inverse kinematic analysis presented in this paper.

As another example, let us consider a case that the target tip position and orientation are unreachable. For simplicity, the target tip position ${ }^{b} \boldsymbol{p}_{t}$ in (88) is reused, while the target orientation 
${ }^{b} \boldsymbol{R}_{t}$ is rotated about ${ }^{b} \boldsymbol{n}$ by 90 degrees. Namely, the target tip position and orientation are

$$
{ }^{b} \boldsymbol{p}_{t}^{d}=\left[\begin{array}{r}
0.035 \\
-0.240 \\
-0.031
\end{array}\right], \quad{ }^{b} \boldsymbol{R}_{t}^{d}=\left[\begin{array}{ccc}
0 & 0 & -1 \\
0 & -1 & 0 \\
-1 & 0 & 0
\end{array}\right]
$$

Since the DOF of the manipulator is five, it can be verified that these position and orientation are not reachable. ${ }^{13}$ Also, only the target orientation is changed about the unconstrained axis by 90 degrees, the expected inverse solution set is the joint angles $\boldsymbol{\theta}$ given by (87) and $\phi=90$ degrees. Solving the inverse kinematics for (89), we obtain four sets of solutions:

$$
\begin{aligned}
& {[\boldsymbol{\theta} \mid \phi]^{T}(\operatorname{deg})=}
\end{aligned}
$$

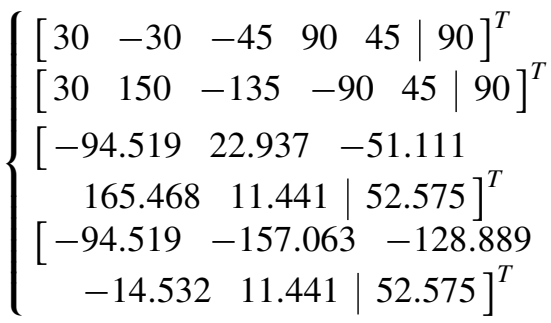

As shown, the first solution set agrees with the expected one. Thus, the inverse kinematic computation method proposed in this paper can properly deal with unreachable target position and orientation.

\section{Discussion and Summary}

\subsection{Extension and limitation of inverse kinematic solving method}

This paper presented an analytical approach to solving the inverse kinematics of the 5-DOF manipulator depicted in Fig. 3. The approach itself can be applied to other manipulators if three consecutive revolute joint axes intersect at a point. This is because existence of such joints enables us to decouple the inverse kinematic problem into the inverse position and orientation problems. It should be noted that such three consecutive joints usually exist for an anthropomorphic manipulator. Therefore, the method proposed in this paper will be useful for other types of anthropomorphic manipulators.

However, intersecting three consecutive joint axes at a point is not the necessary condition for the inverse kinematic problem to be solvable. It is shown in ref. [11] that the inverse kinematics is solvable for a general 5-DOF manipulator if two consecutive revolute joint axes intersect at a point. For such a 5-DOF manipulator, the inverse kinematic problem will be decoupled into the inverse position problem involving four variables and the inverse orientation problem involving two variables. Hence, the approach proposed in this paper is not directly applicable to this case. Nevertheless, if the solutions to the inverse position problem are given as functions of $\phi$, the inverse orientation problem may be solvable. It is shown in ref. [26] that similar approach works well for a redundant manipulator with a wrist. But, this issue has not been addressed yet, and further research will be required.

\subsection{Summary of this paper}

This paper proposed an analytical methodology of solving the inverse kinematic problem for a 5DOF manipulator. The inverse kinematic problem was formulated in a generic form by defining the unconstrained direction and rotation angle $\phi$. Owing to the formulation, the target position and orientation are achieved except an orientation about the user-defined axis, which is fixed with reference to the environment. This paper also presented the singularity analysis. It revealed that there are three kinematic singularities and one mathematical singularity. Finally, the validity of the proposed method was confirmed by numerical examples. 


\section{References}

1. C. Wampler and A. Morgan, "Solving the $6 \mathrm{R}$ inverse position problem using a generic-case solution methodology," Mech. Mach. Theory 26(1), 91-106 (1991).

2. M. Raghavan and B. Roth, "Inverse kinematics for the general 6R manipulator and related linkages," Trans. ASME J. Mech. Des. 115(3), 502-508 (1993).

3. D. Kohli and M. Osvatic, "Inverse kinematics of general 6R and 5R,P serial manipulators," Trans. ASME J. Mech. Des. 115(4), 922-931 (1993).

4. D. Manocha and J. F. Canny, "Efficient inverse kinematics for general 6R manipulators," IEEE Trans. Robot. Autom. 10(5), 648-657 (1994).

5. H. Y. Lee and C. F. Reinholtz, "Inverse kinematics of serial-chain manipulators," Trans. ASME J. Mech. Des. 118(3), 396-404 (1996).

6. S. Z. Xin, L. Y. Feng, H. L. Bing and Y. T. Li, "A simple method for inverse kinematic analysis of the general 6R serial robot,” Trans. ASME J. Mech. Des. 129(8), pp. 793-798 (2007).

7. K. Sugimoto and J. Duffy, "Analysis of five-degree-of-freedom robot arms," Trans. ASME J. Mech. Transmiss. Automn Des. 105(1), 23-27 (1983).

8. L. W. Tsai and A. P. Morgan, "Solving the kinematics of the most general six- and five-degree-of-freedom manipulators by continuation methods," Trans. ASME J. Mech. Transmiss. Automn Des. 107(2), 189-200 (1985).

9. J. Angeles, "Iterative kinematic inversion of general five-axis robot manipulators," Int. J. Robot. Res. 4(4), 59-70 (1986).

10. R. Manseur and K. L. Doty, "A complete kinematic analysis of four-revolute-axis robot manipulators," Mech. Mach. Theory 27(5), 575-586 (1992).

11. R. Manseur and K. L. Doty, "Fast inverse kinematics of five-revolute-axis robot manipulators," Mech. Mach. Theory 27(5), 587-597 (1992).

12. Y. B. Zhou, R. O. Buchal and R. G. Fenton, "Analysis of the general 4R and 5 R robots using a vector algebraic approach," Mech. Mach. Theory 30(3), 421-432 (1995).

13. Y. B. Zhou and F. F. Xi, "Exact kinematic analysis of the general 5R robot," Mech. Mach. Theory 33(1/2), 175-184 (1998).

14. I. M. Chen and Y. Gao, "Closed-Form Inverse Kinematics Solver for Reconfigurable Robots," Proceedings of the 2001 IEEE International Conference Robotics and Automation, vol. 3 (2001) pp. 2395-2400.

15. P. K. Goel, "The Inverse Kinematics Solution, Closed-Form Dynamics and Simulation of Adeptone Industrial Robot," Proceedings of the 1988 IEEE International Conference Robotics and Automation, vol. 3 (1988) pp. 1688-1693.

16. J. Q. Gan, E. Oyama, E. M. Rosales and H. Hu, "A complete analytical solution to the inverse kinematics of the pioneer 2 robotic arm," Robotica 23(1), 123-129 (2005).

17. D. Xu, C. A. Acosta Calderon, J. Q. Gan and H. Hu, "An analysis of the inverse kinematics for a 5-DOF manipulator," Int. J. Autom. Comput. 2(2), 114-124 (2005).

18. J. G. Ramírez-Torres, G. Toscano-Pulido, A. Ramírez-Saldívar and A. Hernández-Ramírez, "A Complete Closed-Form Solution to the Inverse Kinematics Problem for the P2Arm Manipulator Robot," Proceedings of the 2010 Electronics, Robotics and Automotive Mechanics Conf. (2010) pp. 372-377.

19. H. B. Wang and T. Ishimatsu, "Kinematics of a five degree-of-freedom prosthetic arm," Mech. Mach. Theory 33(7), 895-908 (1998).

20. H. Wang, "Kinematics and Control for a Personal Robot with Five Degrees-of-Freedom Arms," Proceedings of the 2007 IEEE International Conference Networking, Sensing and Control (2007) pp. 507-512.

21. F. Sato, T. Nishii, J. Takahashi, Y. Yoshida, M. Mitsuhashi and D. Nenchev, "Experimental Evaluation of a Trajectory/Force Tracking Controller for a Humanoid Robot Cleaning a Vertical Surface," Proceedings of the 2011 IEEE/RSJ International Conference Intelligent Robots and Systems (2011) pp. 3179-3184.

22. R. N. Jazar, Theory of Applied Robotics: Kinematics, Dynamics, and Control, 2nd ed. (Springer, New York, Dordrecht, Heidelberg, London, 2010).

23. J. M. McCarthy, An Introduction to Theoretical Kinematics (The MIT Press, Cambridge, MA, London, 1990).

24. The Mathematical Society of Japan, Encyclopedic Dictionary of Mathematics, 2nd ed. (The MIT Press, Cambridge, MA, London, 1993).

25. N. S. Bedrossian, "Classification of Singular Configurations for Redundant Manipulators," Proceedings of the 1990 IEEE International Conference Robotics and Automation (1990) pp. 818-823.

26. M. Shimizu, H. Kakuya, W. K. Yoon, K. Kitagaki and K. Kosuge, "Analytical inverse kinematic computation for 7-DOF redundant manipulators with joint limits and its application to redundancy resolution," IEEE Trans. Robot. 24(5), 1131-1142 (2008).

\section{Appendix A}

The first-order trigonometric equation in terms of $\theta$ can be represented generally by

$$
\boldsymbol{x}^{T} \boldsymbol{q}=y
$$


where $\boldsymbol{x} \in \mathfrak{R}^{2}$ is a constant vector, $y \in \Re$ is a constant scalar, and $\boldsymbol{q}=[\cos \theta \sin \theta]^{T}$. If $\boldsymbol{q}$ is given, $\theta$ is uniquely determined by using the arctangent function with quadrant information. Hence, it is enough to derive $\boldsymbol{q}$ from (90). Since $|\boldsymbol{q}|=1$,

$$
\boldsymbol{x}^{T} \boldsymbol{q}=|\boldsymbol{x}| \cos \mu=y
$$

where $\mu$ is the angle between $\boldsymbol{x}$ and $\boldsymbol{q}$. When the angle $\mu$ is given, $\boldsymbol{q}$ is described by

$$
\boldsymbol{q}=\frac{1}{\sqrt{\boldsymbol{x}^{T} \boldsymbol{x}}}\left[\cos \mu \boldsymbol{I}_{2}+\sin \mu \boldsymbol{H}\right] \boldsymbol{x}
$$

where $\boldsymbol{I}_{2} \in \mathfrak{R}^{2 \times 2}$ is the identity matrix, and $\boldsymbol{H} \in \mathfrak{R}^{2 \times 2}$ is a constant matrix given by

$$
\boldsymbol{H}=\left[\begin{array}{cc}
0 & -1 \\
1 & 0
\end{array}\right]
$$

From (91),

$$
\begin{aligned}
& \cos \mu=\frac{y}{\sqrt{\boldsymbol{x}^{T} \boldsymbol{x}}} \\
& \sin \mu= \pm \sqrt{1-\cos ^{2} \mu}= \pm \frac{1}{\sqrt{\boldsymbol{x}^{T} \boldsymbol{x}}} \sqrt{\boldsymbol{x}^{T} \boldsymbol{x}-y^{2}}
\end{aligned}
$$

Substituting them into (92) gives

$$
\boldsymbol{q}=\frac{1}{\boldsymbol{x}^{T} \boldsymbol{x}}\left[y \boldsymbol{I}_{2} \pm \sqrt{\boldsymbol{x}^{T} \boldsymbol{x}-y^{2}} \cdot \boldsymbol{H}\right] \boldsymbol{x} .
$$

Note that the quantity under the square root must be nonnegative. This provides the existence condition of the solution:

$$
y^{2} \leq \boldsymbol{x}^{T} \boldsymbol{x} .
$$

\section{Appendix B}

Let $w_{i j}$ be the $(i, j)$ element of $\boldsymbol{W}$. Then,

$$
\boldsymbol{W}^{T} \boldsymbol{W}=\left[\begin{array}{cc}
w_{11}^{2}+w_{21}^{2} & w_{11} w_{12}+w_{21} w_{22} \\
w_{11} w_{12}+w_{21} w_{22} & w_{12}^{2}+w_{22}^{2}
\end{array}\right]
$$

Since $\boldsymbol{W}^{T} \boldsymbol{W}$ is positive definite,

$$
\begin{aligned}
\operatorname{trace} \boldsymbol{W}^{T} \boldsymbol{W} & =\lambda_{1}+\lambda_{2} \\
\operatorname{det} \boldsymbol{W}^{T} \boldsymbol{W} & =\lambda_{1} \lambda_{2} .
\end{aligned}
$$

From these equations, the eigenvalues $\lambda_{1}$ and $\lambda_{2}$ can be obtained as

$$
\begin{aligned}
& \lambda_{1}=\frac{t-\sqrt{t^{2}-4 d}}{2} \\
& \lambda_{2}=\frac{t+\sqrt{t^{2}-4 d}}{2}
\end{aligned}
$$

where $t=\operatorname{trace} \boldsymbol{W}^{T} \boldsymbol{W}=w_{11}^{2}+w_{21}^{2}+w_{12}^{2}+w_{22}^{2}$ and $d=\operatorname{det} \boldsymbol{W}^{T} \boldsymbol{W}=\left(w_{11} w_{22}-w_{12} w_{21}\right)^{2}$. Note that $t^{2} \geq 4 d$ always holds, hence real eigenvalues always exist. 
Since $\boldsymbol{U}$ in (52) is orthonormal, it can be represented by

$$
\boldsymbol{U}=\left[\begin{array}{ll}
\boldsymbol{u}_{1} & \boldsymbol{u}_{2}
\end{array}\right]=\left[\begin{array}{cc}
\cos v & -\sin v \\
\sin v & \cos v
\end{array}\right]
$$

where $v$ is a real scalar. To satisfy $\boldsymbol{W}^{T} \boldsymbol{W}=\boldsymbol{U} \boldsymbol{\Lambda} \boldsymbol{U}^{T}, v$ must ensure the relations

$$
\begin{aligned}
& \left(\lambda_{1}-\lambda_{2}\right) \cos 2 v=w_{11}^{2}+w_{21}^{2}-w_{12}^{2}-w_{22}^{2} \\
& \left(\lambda_{1}-\lambda_{2}\right) \sin 2 v=2\left(w_{11} w_{12}+w_{21} w_{22}\right) .
\end{aligned}
$$

From these equations, $v$ is uniquely determined. Substituting it into (98), the eigenvectors $\boldsymbol{u}_{1}$ and $\boldsymbol{u}_{2}$ are obtained. Note that, if $\lambda_{1}=\lambda_{2} \equiv \lambda, v$ is arbitrary, because $\boldsymbol{W}^{T} \boldsymbol{W}=\lambda \boldsymbol{I}_{2}$. 